\title{
A tale of two STs: molecular and clinical epidemiology of MRSA t304 in Norway 2008-2016
}

\author{
Hege Enger ${ }^{1}$ (D) Kjersti Wik Larssen ${ }^{1} \cdot$ Elise Størvold Damås ${ }^{1} \cdot$ Hege Vangstein Aamot $^{2} \cdot$ Anita Blomfeldt $^{2}$ (i) . \\ Petter Elstrøm ${ }^{3}{ }^{-} \cdot$ Christina Gabrielsen Ås ${ }^{1,4}$
}

Received: 22 June 2021 / Accepted: 23 September 2021 / Published online: 23 October 2021

(c) The Author(s) 2021

\begin{abstract}
The purpose of this study was to investigate the epidemiological, molecular, and clinical characteristics of MRSA t304/ST8 and t304/ST6 in Norway from 2008 to 2016. Clinical and epidemiological data were collected for each case included in the study. Strains were characterized by PCR, spa typing, antimicrobial susceptibility testing, and whole genome sequencing. The overall number of cases of MRSA t304 increased from 27 in 2008 to 203 in 2016. Most MRSA t304/ST8 cases were defined as HA-MRSA (89.9\%) and diagnosed in persons with Norwegian background, many of them living in nursing homes $(62.3 \%)$. The number of $\mathrm{t} 304 / \mathrm{ST} 8$ cases declined throughout the study period and it has not been reported in Norway since 2014. The increasing MRSA t304/ST6 genotype has mainly been introduced to Norway by immigration from the Middle East, but also from other parts of the world. The t304/ST6 clone is mostly classified as CA-MRSA (75.1\%), does not seem to cause serious infections, is not multi-resistant, and has not yet caused outbreaks in Norway. This study provides an example of two MRSA clones with the same spa type found in different epidemiological settings. This is very unusual, but still a reminder that spa typing in some cases may have insufficient discriminatory power for surveillance of MRSA. Our results highlight the importance of active surveillance and characterization of emerging MRSA clones with high potential for spread in the community, which may potentially cause outbreaks in healthcare facilities.
\end{abstract}

Keywords MRSA $\cdot$ t304/ST6 $\cdot$ t304/ST8 $\cdot$ Surveillance $\cdot$ Genotyping $\cdot$ Epidemiology

\section{Introduction}

Staphylococcus aureus causes a wide spectrum of infections ranging from asymptomatic carriage to skin and soft tissue infections, bone and joint infections, bacteraemia, and endocarditis. Methicillin-resistant S. aureus (MRSA) possesses resistance against the bactericidal beta-lactam group

Christina Gabrielsen Ås

christina.gabrielsen@stolav.no

1 The Norwegian MRSA Reference Laboratory, Department of Medical Microbiology, St. Olavs Hospital, Trondheim University Hospital, Trondheim, Norway

2 Department of Microbiology and Infection Control, Akershus University Hospital, Lørenskog, Norway

3 Department of Antibiotic Resistance and Infection Prevention, Norwegian Institute of Public Health, Oslo, Norway

4 Department of Clinical and Molecular Medicine, Norwegian University of Science and Technology, Trondheim, Norway of antibiotics, which is the cornerstone of treatment for staphylococcal infections. The incidence of MRSA varies in different parts of the world, but the total disease burden and mortality is considerable. A European study estimated a total of 33,000 deaths due to infections with resistant microbes in the EU and the European Economic Area in 2015, with MRSA contributing to a substantial part of the mortality and morbidity [1].

The incidence of MRSA in Norway is low compared to many other countries, with 49 per 100,000 person years in 2016 [2]. The proportion of MRSA in both S. aureus bloodstream infections (BSI) and wound infections has been stable at around $1 \%$ the last decade in Norway [2, 3]. However, from 2008 to 2016, the total number of reported MRSA infections and carriage strains in Norway has gradually increased from 652 to 2538 cases per year [2,3]. One of the genotypes that has contributed most to this increase, both in Norway and in the other Nordic countries, is MRSA spa type t304 [4]. MRSA t304/ST8 was reported as the cause of several nursing home-related outbreaks in South East 
Norway from 2005 to 2011 [5]. This genotype has also been described in Martinique in the Caribbean [6].

In 2015, a study describing a prolonged neonatal wardrelated outbreak in Copenhagen caused by MRSA t304/ST6 was published [7], and the same year, this genotype was detected by the Norwegian MRSA reference laboratory. MRSA t304/ST6 had previously been reported as a dominating genotype in Oman [8], and had been detected in the United Arab Emirates [9], as well as in $c f r$-positive MRSA strains from pigs in China [10].

In this study, we wanted to explore and compare these two genotypes (MRSA t304/ST6 and t304/ST8) with regard to epidemiological, molecular, and clinical characteristics, in an attempt to elucidate the origins of these genotypes, possible adaptations to healthcare and community settings, and whether MRSA t304/ST6 may represent a high risk clone that is becoming dominant in Norway.

\section{Materials and Methods}

\section{Study design and population}

All laboratory-confirmed cases of MRSA in Norway, including both carriage and infections, are notifiable to the Norwegian Surveillance System for Communicable Diseases (MSIS), and all new MRSA strains are sent to the Norwegian MRSA reference laboratory for confirmation, genotyping, and biobank storage. All cases of MRSA t304 in Norway from 2008 to 2016 were included, one strain per individual that had given passive informed consent to participate in the study. After exclusion of persons without available contact information $(n=79), 318$ cases were included in the study.

\section{Epidemiological data}

Epidemiological data on all included cases were collected from the Norwegian Surveillance System for Communicable Diseases (MSIS), the Norwegian National Population Registry, the Norwegian Healthcare worker registry (HPR), and from the requisition filled out by the treating physicians. The HPR contains information about all persons educated as healthcare workers (HCWs). Information from MSIS includes age, sex, birth country of the person and the person's parents, admission to hospital or nursing home, and travel history, in addition to information about the bacterial strain. In this study, due to lack of data on admission time for hospitalized patients, we used a broad definition of healthcare-associated MRSA (HA-MRSA). This included MRSA diagnosed in hospitals or nursing homes and/or MRSA diagnosed in HCWs, while community-acquired MRSA (CAMRSA) was defined as all other cases. Statistical analyses of discrete variables were performed using Fisher's exact test, while age distributions were compared with Welch's $t$-test. The Benjamini-Hochberg method was used to correct for multiple hypothesis testing, with adjusted $p$-values $<0.05$ regarded as statistically significant.

\section{Bacterial strains and antibiotic susceptibility testing}

Bacterial strains were cultured on blood agar plates at $35^{\circ} \mathrm{C}$, if poor growth incubated with $5 \% \mathrm{CO}_{2}$. Susceptibility testing was performed on all strains using the EUCAST (European Committee on Antimicrobial Susceptibility Testing) disk diffusion method and categorized as either susceptible, intermediate, or resistant according to the 2016 EUCAST breakpoints. For clindamycin, inducible resistance was recorded as described in the EUCAST expert rules. For vancomycin, gradient strip test was used. For strains from 2008 to 2015, susceptibility testing was performed by the reference laboratory using disks from Oxoid and gradient strip tests from Biomerieux and Liofilchem. From 2016, susceptibility testing was performed by the referring laboratories, and results were reported to the reference laboratory.

\section{DNA extraction, confirmation PCR and spa typing}

Extraction of genomic DNA was routinely performed by heat lysis. Briefly, a few colonies from pure culture were suspended in molecular grade water and heated to $95{ }^{\circ} \mathrm{C}$ for $15 \mathrm{~min}$ with shaking (300 rpm). After centrifugation at $14,500 \mathrm{rpm}$ for $2 \mathrm{~min}$, the supernatant was extracted and used for PCR analyses.

Confirmation of all MRSA strains was performed using a multiplex conventional PCR detecting the $m e c A$ gene, the S. aureus-specific spa gene, and the Panton-Valentine leukocidin (PVL) genes followed by gel electrophoresis [11].

All received strains were genotyped by spa typing, sequencing of the polymorphic region $\mathrm{X}$ of the $S$. aureus specific spa gene according to Harmsen et al. [12] using primers $s p a-1113 \mathrm{f}$ and $s p a-1514 \mathrm{r}$ [13]. The sequences obtained were assigned to spa types using the Ridom Spa Server [14].

\section{Discriminatory high-resolution melt-PCR}

To enable rapid differentiation between MRSA t304/ST6 and t304/ST8, a high-resolution melt-PCR (HRM-PCR) was established based on a discriminatory single nucleotide polymorphism (SNP) in position 294 the phosphate acetyltransferase ( $p t a)$ allele. Real-time PCR was performed with forward and reverse primers (5'-AAGCAGATGGTTTAG TTAGT-3' and 5'-ATACACCTGGTTTCGTTT-3' accordingly) by a two-step PCR program on a CFX96 Real-time 
Fig. 1 Temporal distribution of MRSA t304/ST8 $(n=75)$ and t304/ST6 ( $n=400)$ cases (bars) and as a percentage of total MRSA cases in Norway (lines)

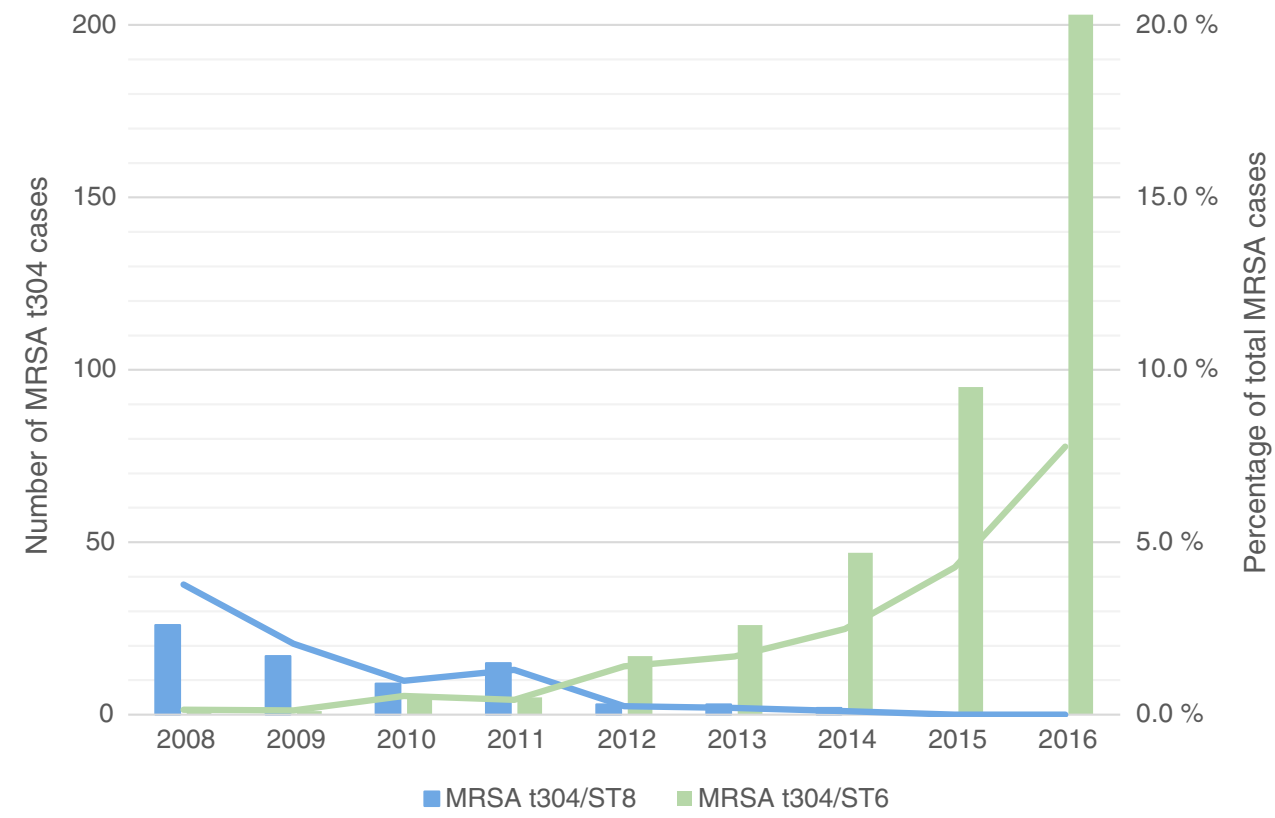

system (Bio-Rad). HRM analysis was performed using the Precision Melt Analysis ${ }^{\mathrm{TM}}$ Software.

\section{Whole genome sequencing and bioinformatics analyses}

Whole genome sequencing (WGS) was performed on a subset of randomly selected strains from each year of the study period, maximum one strain from each household, including $20 \mathrm{t} 304 / \mathrm{ST} 8$ and $88 \mathrm{t} 304 / \mathrm{ST} 6$. This covered $43.9 \%$ and $48.6 \%$ of all households of ST8 and ST6 cases accordingly.

For WGS, cells were first treated with proteinase $\mathrm{K}(2 \mathrm{mg} /$ $\mathrm{mL})$ and lysostaphin $(0.1 \mathrm{mg} / \mathrm{mL})$ for $15 \mathrm{~min}$ with shaking at $37^{\circ} \mathrm{C}$, before heating for $15 \mathrm{~min}$ at $65^{\circ} \mathrm{C}$. Genomic DNA was then isolated using the EZ1 DNA tissue kit with an EZ1 Advanced XL instrument (Qiagen). Sequencing libraries were prepared using the Nextera XT sample prep kit and sequenced on the MiSeq platform with MiSeq v3 reagents, with 300 bp paired end reads (Illumina).

Raw data were quality controlled, trimmed/filtered, and de novo assembled, and the assembled genomes annotated, typed (multi-locus sequence typing (MLST)), and characterized (resistance and virulence genes) with the Nullarbor pipeline version 2.0 [15]. Resistance and virulence genes were identified using the NCBI National Database of Antibiotic Resistant Organisms (NDARO) [16] and the Virulence Factor DataBase (VFDB) accordingly [17]. Additionally, SCCmecFinder 1.2 was used for SCCmec typing of all strains [18]. The core and accessory genome of the study strains and $15 \mathrm{~S}$. aureus reference genomes was defined and a core genome alignment produced by Roary version 3.13 [19]. Fasttree 2.1.10 [20] was used to infer a maximum likelihood core genome phylogeny with the GTR model, and distance estimation was performed by Molecular Evolutionary Genetics Analysis (MEGA) software [21]. Genetic distances in general refer to the number of SNPs in a $S$. aureus core genome alignment of 1,280,334 bp. Visualizations of phylogenies with metadata were created using iTOL [22].

\section{Results}

\section{Temporal distribution}

A total of 475 MRSA t304 strains were received by the Norwegian MRSA reference laboratory in the 9 year study period (2008-2016), with an overall increase in cases from 27 in 2008 to 203 in 2016. Results from HRM-PCR-based assignment to ST8 or ST6 show that the number of cases of MRSA t304/ST8 was high in the initial years of the study period (Fig. 1), but decreased steadily until 2011. The situation was reversed for MRSA t304/ST6, with a sharp increase in cases from 2008 to 2016 (Fig. 1). Of the total MRSA t304 strains, 318 (66.8\%) were included for further analyses.

\section{Epidemiology}

The epidemiological data revealed a significant distinction between the age distributions of the MRSA t304/ST8 and MRSA t304/ST6 strains (Table 1, Fig. 2). The average age was 73 years (range 23-97 years) for persons with t304/ST8, compared to 26 years (range $0-85$ years) for t304/ST6. In 
Table 1 Clinical and epidemiological characteristics of MRSA t304 cases

\begin{tabular}{|c|c|c|c|c|c|c|}
\hline & \multirow[t]{2}{*}{ Category } & \multicolumn{2}{|c|}{ t304/ST8 } & \multicolumn{2}{|c|}{ t304/ST6 } & \multirow[t]{2}{*}{$P$-value ${ }^{3}$} \\
\hline & & $\mathbf{n}$ & $\%$ & $\mathbf{n}$ & $\%$ & \\
\hline Total & Cases & 69 & 21.7 & 249 & 78.3 & \\
\hline \multirow[t]{4}{*}{ Age and sex } & Age, average & 73 & & 26 & & \\
\hline & Age, range & $23-9$ & & $0-85$ & & $1.76 \mathrm{E}-33$ \\
\hline & Fraction of men & 24 & 34.8 & 125 & 50.2 & 4.7E-02 \\
\hline & Fraction of women & 45 & 65.2 & 124 & 49.8 & \\
\hline \multirow[t]{9}{*}{ Birth country } & Norway & 57 & 82.6 & 96 & 38.6 & 2.7E-11 \\
\hline & Norwegian heritage & 57 & 100.0 & 50 & 52.1 & 6.6E-12 \\
\hline & Non-Norwegian heritage ${ }^{1}$ & 0 & 0.0 & 46 & 18.5 & \\
\hline & Non-Norwegian birth country & 11 & 15.9 & 153 & 61.4 & 2.7E-11 \\
\hline & Africa except Egypt & 2 & 18.2 & 24 & 15.7 & $1.2 E-04$ \\
\hline & Asia except Middle East ${ }^{2}$ & 5 & 45.5 & 38 & 24.8 & \\
\hline & Europe except Norway & 4 & 36.4 & 8 & 5.2 & \\
\hline & Middle East & 0 & 0.0 & 81 & 52.9 & \\
\hline & Unknown/other & 1 & 9.1 & 2 & 1.3 & \\
\hline \multirow[t]{7}{*}{ MRSA acquired } & Norway & 51 & 73.9 & 42 & 16.9 & 3.8E-21 \\
\hline & Abroad & 1 & 1.4 & 115 & 46.2 & \\
\hline & Work-related & 0 & 0.0 & 3 & 2.6 & $1.6 \mathrm{E}-01$ \\
\hline & Home country visit & 0 & 0.0 & 31 & 27.0 & \\
\hline & Tourism & 1 & 100.0 & 8 & 7.0 & \\
\hline & Immigration & 0 & 0.0 & 65 & 56.5 & \\
\hline & Unknown & 17 & 24.6 & 92 & 36.9 & \\
\hline \multirow[t]{4}{*}{ Carriage/infection } & Carriage & 51 & 73.9 & 188 & 75.5 & $9.5 \mathrm{E}-01$ \\
\hline & Infections & 18 & 26.1 & 61 & 24.5 & \\
\hline & Wound/pus & 16 & 88.9 & 49 & 80.3 & $6.0 \mathrm{E}-01$ \\
\hline & Other infections & 2 & 11.1 & 12 & 19.7 & \\
\hline \multirow[t]{2}{*}{ Outbreaks } & Outbreak-related cases & 46 & 66.7 & 2 & 0.8 & $1.9 E-34$ \\
\hline & Non-outbreak-related cases & 23 & 33.3 & 247 & 99.2 & \\
\hline \multirow[t]{6}{*}{ HA/CA } & HA-MRSA & 62 & 89.9 & 62 & 24.9 & $1.3 \mathrm{E}-22$ \\
\hline & Infections & 15 & 24.2 & 15 & 24.2 & $1.0 \mathrm{E}+00$ \\
\hline & Carriage & 47 & 75.8 & 47 & 75.8 & \\
\hline & CA-MRSA & 7 & 10.1 & 187 & 75.1 & $1.3 E-22$ \\
\hline & Infections & 3 & 42.9 & 46 & 24.6 & $4.8 \mathrm{E}-01$ \\
\hline & Carriage & 4 & 57.1 & 141 & 75.4 & \\
\hline
\end{tabular}

${ }^{1}$ Defined as a case where at least one parent was born abroad

${ }^{2}$ Definition of the Middle Eastern Countries according to World Population Review: Bahrain, Cyprus, Egypt, Iran, Iraq, Israel, Jordan, Kuwait, Lebanon, Oman, Palestine, Qatar, Saudi Arabia, Syria, Turkey, The United Arab Emirates, and Yemen

${ }^{3}$ Corrected $p$-values, significant values displayed in bold. For birth country, the $p$-value corresponds to comparison between Norway and non-Norwegian birth country; for HA/CA, the given $p$-value corresponds to comparison between HA-MRSA and CA-MRSA the $\mathrm{t} 304 / \mathrm{ST} 8$ group, there were more women $(65.2 \%)$ than men $(34.8 \%)$.

The registered place of acquisition for MRSA cases also differed distinctly between the ST8 and ST6 groups (Table 1). One single case of MRSA t304/ST8 was reported as contracted abroad; the corresponding number for ST6 cases was $115 / 249$ (46.2\%). Among these, a majority were associated with either immigration $(65 / 115,56.5 \%)$ or home country visit $(31 / 115,27.0 \%)$. Only 8 ST6 cases were connected to tourism, and 3 were work-related.

There was a significant difference between the ST8 and ST6 groups in terms of association to birth country (Table 1, Fig. 3). In the ST8 group, 57/69 (82.6\%) persons were born in Norway to Norwegian parents. In the ST6 group, 153 (61.4\%) were born abroad. Of these, 81 (52.9\%) were born in Middle Eastern countries, most frequently in Syria and Iraq, while 38/153 (24.8\%) persons 
Fig. 2 Age distribution of patients with MRSA t304/ST8 $(n=69)$ and t304/ST6 $(n=249)$

Fig. 3 Map displaying birth country for patients with MRSA t304/ST8 (blue) and t304/ST6 (green), with circles sized relative to the number of cases in each country
70
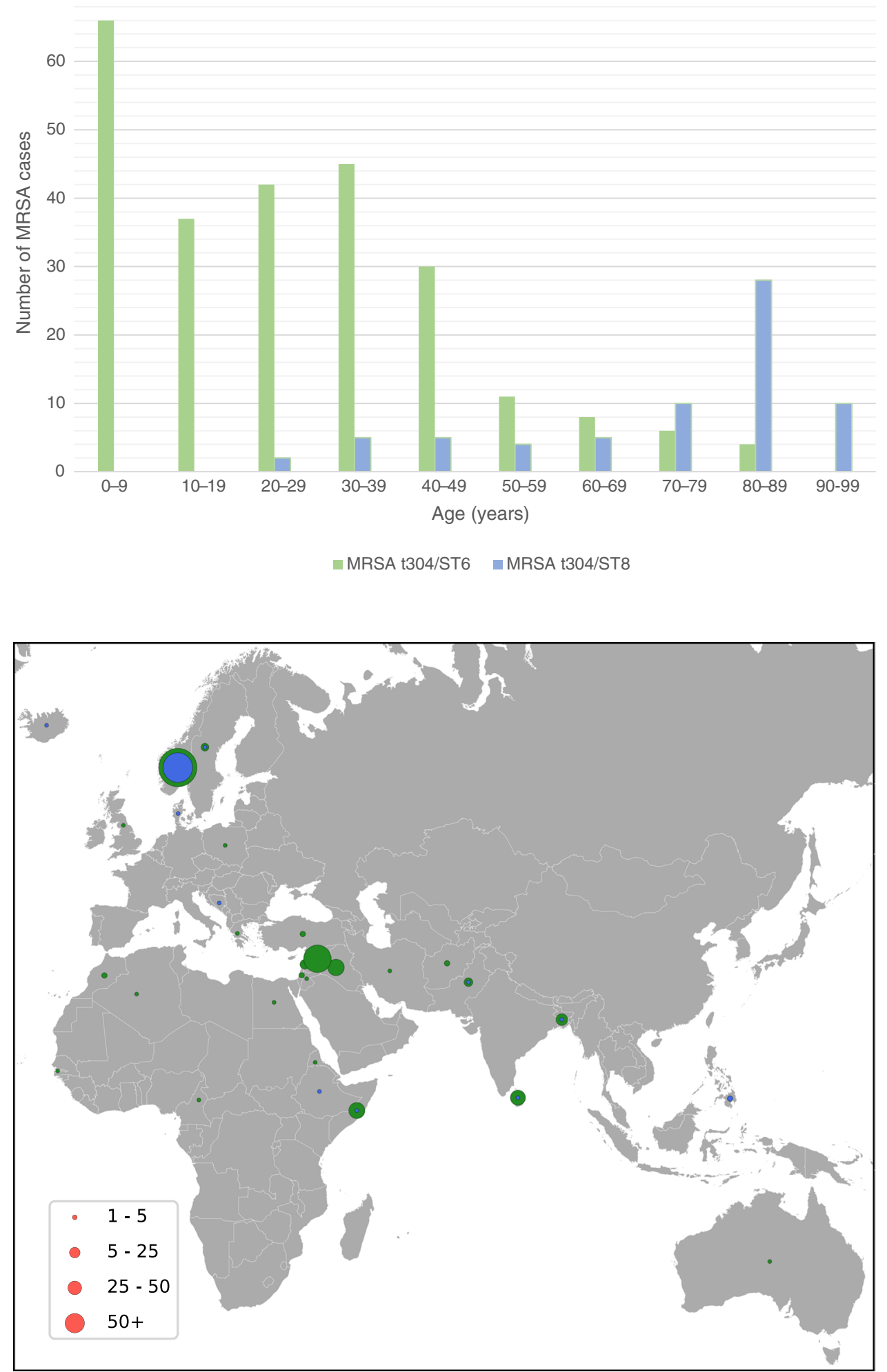

were born in other Asian countries. Forty-six persons $(18.5 \%)$ with ST6 had non-Norwegian heritage with at least one parent born abroad, 41 of these 46 were children from 0 to 18 years.

\section{Clinical characteristics}

The infection rates of MRSA t304/ST8 and t304/ST6 were similar, $26.1 \%$ in the ST8 group and $24.5 \%$ in the ST6 group (Table 1). Wound infections and abscesses were the 


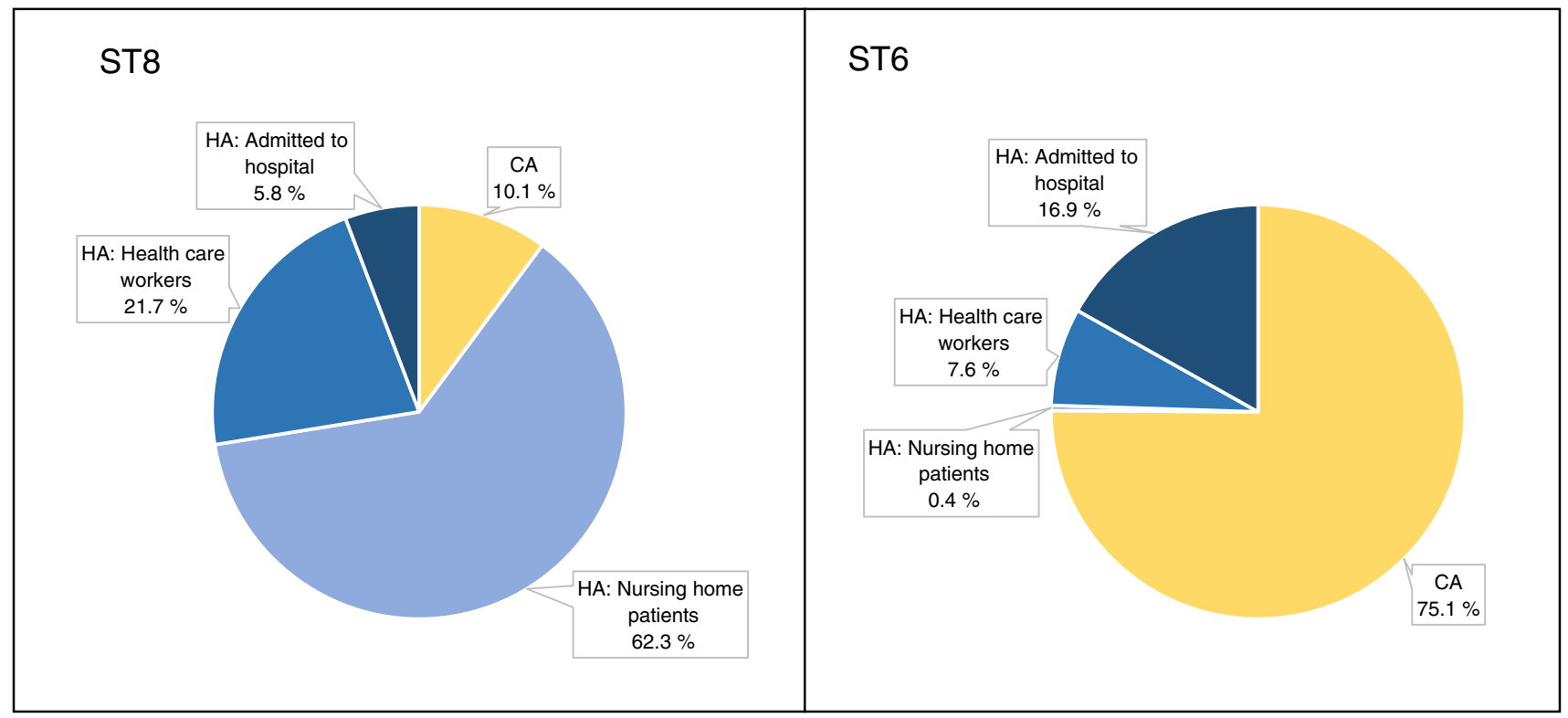

Fig. 4 Epidemiological characteristics of MRSA t304 cases. Comparison of cases defined as community-associated or healthcare-associated for t304/ST8 and t304/ST6 cases

dominating causes of infections in both groups, causing $88.9 \%$ of the infections in the ST8 group and $80.3 \%$ in the ST6 group. Other infections ( $n=14$ for ST8 and ST6) included bacteremia, pneumonia, genital infection, mastitis, media otitis, conjunctivitis, and respiratory infection of unknown significance. Only 14/318 (4.4\%) persons with infections were admitted to hospital at the time the MRSA strains were collected.

In total, 62 of the 69 (89.9\%) t304/ST8 cases were defined as HA-MRSA (Table 1). This included $43(62.3 \%)$ persons living in a nursing home, 15 (21.7\%) HCWs, and 4 (5.8\%) persons admitted to a hospital (Fig. 4). The infection rate within $\mathrm{t} 304 / \mathrm{ST} 8$ HA-MRSA was $24.2 \%$.

Sixty-two (24.9\%) of the t304/ST6 cases were defined as HA-MRSA (Fig. 4). Only one person lived in a nursing home, 19 (7.6\%) were HCWs, and 42 (16.9\%) were admitted to hospital. CA-MRSA accounted for $187 / 249$ (75.1\%) of the ST6 group. The infection rate was $24.2 \%$ for HA-MRSA and $24.6 \%$ for CA-MRSA.

Forty-eight $(15.1 \%)$ of the MRSA t304 strains were reported to be outbreak related; almost all these were t304/ ST8 (46/48) (Table 1). The majority of outbreak-related cases included persons living in nursing homes $(35 / 48$, $72.9 \%$ ) belonging to 6 different outbreaks, where the largest outbreak included 21 persons.

\section{Antimicrobial susceptibility}

Both sequence types of MRSA t304 displayed limited antimicrobial resistance, as $59.1 \%$ of all strains were resistant only to cefoxitin/betalactams (Fig. 5). Resistance against two antibiotic groups, including cefoxitin, was detected in 111 strains (34.9\%), 16 strains (5.0\%) were resistant against 3 antibiotic groups, and only 3 strains $(0.9 \%)$ displayed resistance against 4 or 5 different antibiotic groups. The t304/ ST8 group had higher occurrence of tetracyclin resistance $(91.2 \%)$ compared to the t304/ST6 group $(6.0 \%)$.

\section{Resistance and virulence genes}

WGS was performed on $20 \mathrm{t} 304 / \mathrm{ST} 8$ strains and $88 \mathrm{t} 304 /$ ST6 strains. Phylogenetic analysis of the pangenome (Fig. 6) showed that the two groups were clearly distinct lineages, with relatively low core genome SNP distances within each group (median 26 SNPs and 62 SNPs for MRSA t304/ST8 and t304/ST8 accordingly). Three strains had point mutations in MLST alleles (other than pta) causing novel $(n=2)$ or other $(n=1)$ sequences types within CC6. In general, infection strains and carriage strains were dispersed, and there were no clear correlations between specific clusters and country of birth.

Results from in silico resistance gene prediction showed low number of resistance genes for both groups of MRSA t304 (Figure S1). All strains shared mecA, fosB, and tet (38). Among the t304/ST8 group, 18/20 (90.0\%) strains had tetM, 17/20 (85.0\%) blaZ, and 17/20 (85\%) dfrC. The t304/ST6 strains displayed even lower occurrence of resistance genes, with 16/88 (18.2\%) ermC, 5/88 (5.7\%) tet $K$, and 3/88 (3.4\%) blaZ. 


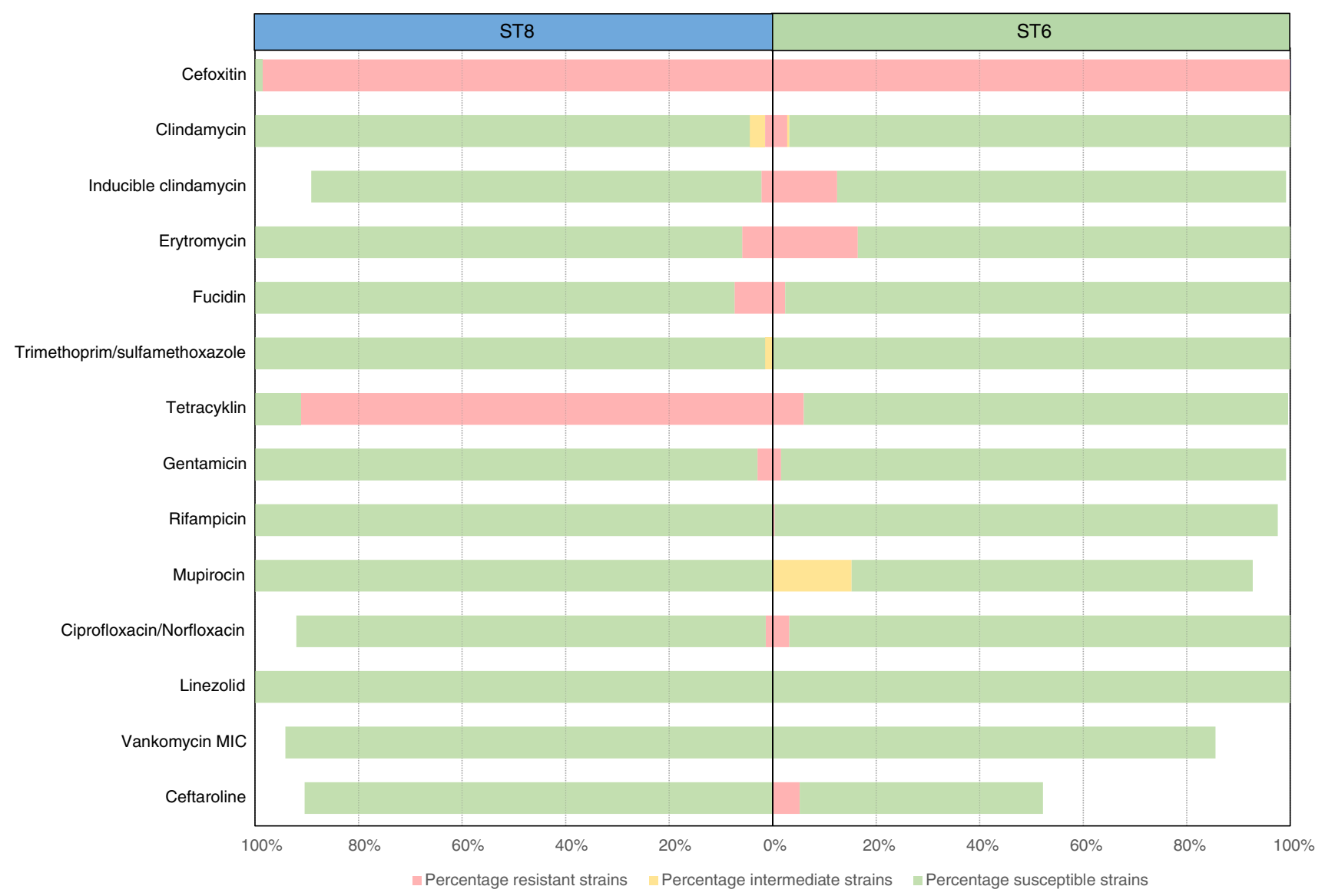

Fig. 5 Phenotypic antimicrobial susceptibility of MRSA t304/ST8 and t304/ST6 strains

There were 72 different virulence factors detected in the sequenced MRSA t304 strains $(n=108)$ (Figure S1), of which 46 were present in all strains and 11 in more than $94 \%$ of strains. On average, 64 and 67 virulence factors were found in t304/ST8 and t304/ST6, respectively. The main differences included a complete serotype 8 capsule gene cluster in t304/ST6, which was not detected in the t304/ST8 strains. Most t304/ST6 strains (90.0\%) encoded staphylococcal enterotoxin D, which was not found in any of the t304/ ST8 strains. Furthermore, the MSCRAMMs encoded by clfB, $c n a, s d r C$, and $s d r E$ were found in a majority of t304/ ST6 strains $(>68.5 \%)$, while only in a minority of t304/ST8 strains $(<10.0 \%)$.

In silico SCCmec typing revealed that SCCmec type IV or IVa dominated in both sequence types, while a small subset of t304/ST6 strains had SCCmec type IVc $(n=6)$ or SCCmec type $\mathrm{V}(n=4)$ (Figure S1).

\section{Discussion}

Two different MRSA t304 clones have been circulating in Norway, detected in different epidemiological settings. While t304/ST8 was already established in Norway before the study period $[4,23]$ and has not been reported since 2014, t304/ST6 has caused a sharp increase of MRSA t304 in recent years. Two different clones sharing the same spa type appears to be a rare event [24], but this study is still a reminder that spa typing may have insufficient discriminatory power for surveillance of MRSA, even in the epidemiologically heterogeneous MRSA situation in Norway.

The MRSA t304/ST8 clone showed a significant association with outbreaks in nursing homes, with many cases among elderly persons and HCWs, which explains the female dominance among the ST8 cases. Due to this, t304/ ST8 was initially designated as healthcare-associated, but our study finds little evidence of this clone being particularly adapted to the HA setting. Rather, based on SCCmec type, minimal antimicrobial resistance as well as high rate of carriage, it displays many typical CA-MRSA hallmarks, except for the absence of PVL. No detected cases of t304/ST8 since 2014 could be an effect of good handling of outbreaks and/ or improved infection control measures in nursing homes during the recent years.

The MRSA t304/ST6 clone was mainly communityassociated, mostly detected in younger people and children. A large proportion of cases were either acquired abroad 


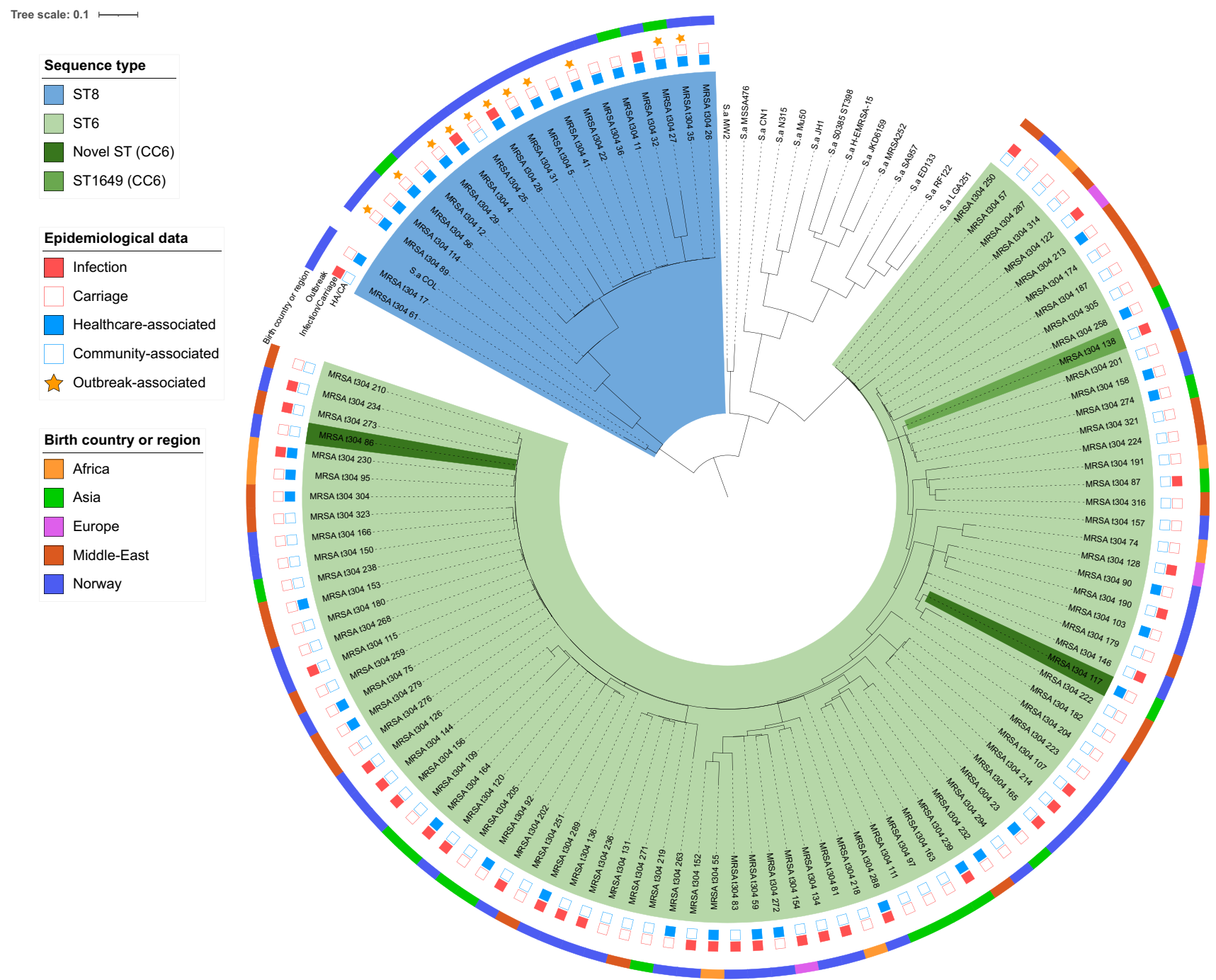

Fig. 6 Pan-genome phylogeny of MRSA t304/ST8 and t304/ST6 with epidemiological data and birth country

following immigration or home-country visit and/or had non-Norwegian heritage or birth country. Only a small number of cases were associated with work-related travel or vacation, indicating that transmission of this clone may require close contact with the local population. A majority of the non-Norwegian born persons with MRSA t304/ ST6 were from Syria and Iraq, indicating a Middle Eastern origin of this clone, as was previously also suggested in a Danish study [25]. The sharp increase of MRSA t304/ST6 in Norway during the study period may partly be a result of increased immigration from these countries due to the refugee crisis which peaked in 2015-2016. MRSA t304/ST6 was also acquired and/or detected in significant numbers in persons born in Africa and other Asian countries, suggesting repeated introductions of this clone to Norway during the study period. Despite this, phylogenetic analysis displayed a surprisingly conserved t304/ST6 clone, with very little variation in terms of resistance and virulence genes.

The limitations of this study include incomplete information from the registers used, for example, on the place of acquisition of MRSA. A significant number of cases did furthermore not have available address information, particularly immigrants, and excluding these persons from the study may have led to an underestimation of the number of imported cases. On the other hand, more comprehensive screening routines among refugees may potentially have contributed to higher numbers of detected MRSA cases in this group. Higher screening rates around HA-related MRSA cases than CA-related cases are also likely, leading to an overestimation of HA-related cases. The broad definition of HA-MRSA used in this study may also be debated, since the place MRSA was diagnosed is not necessarily where MRSA was acquired. Information about the time of admission to 
healthcare institutions would probably have reduced the number of HA-MRSA, providing the ability to exclude MRSA detected within $48 \mathrm{~h}$ of admission.

The main aim of the study was to compare the two different MRSA clones and determine whether MRSA t304/ ST6 represents a high risk clone, which would warrant closer attention in our national surveillance program. Our results, however, show that MRSA t304/ST6 does not appear to be especially virulent, with low levels of antibiotic resistance and mainly associated with carriage in the community. As far as we know, MRSA t304/ST6 has not yet caused outbreaks in Norwegian healthcare institutions. However, this needs to be carefully monitored, since most of the MRSA-positive persons are still young and presumably healthy. If many become persistent carriers of MRSA, this situation could change with time. There is special concern about vulnerable patients in, e.g., neonatal units, where many MRSA outbreaks have taken place the recent years, including one caused by MRSA t304/ST6 [7, 26, 27]. Active surveillance of MRSA and characterization of emerging MRSA clones, as performed in this study, is thus important in order to evaluate and potentially adjust existing MRSA screening and infection control guidelines to prevent MRSA from establishing in the Norwegian healthcare system.

Supplementary Information The online version contains supplementary material available at https://doi.org/10.1007/s10096-021-04353-9.

Acknowledgements The authors would like to thank the Norwegian microbiology laboratories for submitting clinical strains to the Norwegian MRSA reference laboratory at St. Olavs Hospital, as well as the laboratory personnel who have contributed to the analysis of these strains. The HRM-PCR described in this article was developed as part of a bachelor project by Elise Damås and Kristine Gjertsen.

Funding Open access funding provided by NTNU Norwegian University of Science and Technology (incl St. Olavs Hospital - Trondheim University Hospital). This project was supported by funds from the Clinic of Laboratory Medicine, St. Olavs Hospital, Trondheim University Hospital, Trondheim, Norway.

Availability of data and material Due to Norwegian legislation on medical and health research, the full clinical and epidemiological dataset cannot be made openly available as per ethical approval by the Regional Committees for Medical and Health Research Ethics (REC). Sequence data are available from GenBank under BioProject ID PRJNA737487.

\section{Declarations}

Ethics approval The project was approved by the Regional Committees for Medical and Health Research Ethics (REC) Central with reference number 2017/2050

Consent to participate Not applicable.

Consent for publication Not applicable.
Conflict of interest The authors declare no competing interests.

Additional declarations for articles in life science journals that report the results of studies involving humans and/or animals Not applicable.

Open Access This article is licensed under a Creative Commons Attribution 4.0 International License, which permits use, sharing, adaptation, distribution and reproduction in any medium or format, as long as you give appropriate credit to the original author(s) and the source, provide a link to the Creative Commons licence, and indicate if changes were made. The images or other third party material in this article are included in the article's Creative Commons licence, unless indicated otherwise in a credit line to the material. If material is not included in the article's Creative Commons licence and your intended use is not permitted by statutory regulation or exceeds the permitted use, you will need to obtain permission directly from the copyright holder. To view a copy of this licence, visit http://creativecommons.org/licenses/by/4.0/.

\section{References}

1. Cassini A, Hogberg LD, Plachouras D, Quattrocchi A, Hoxha A, Simonsen GS, Colomb-Cotinat M, Kretzschmar ME, Devleesschauwer B, Cecchini M, Ouakrim DA, Oliveira TC, Struelens MJ, Suetens C, Monnet DL, Burden of AMRCG, (2019) Attributable deaths and disability-adjusted life-years caused by infections with antibiotic-resistant bacteria in the EU and the European Economic Area in 2015: a population-level modelling analysis. Lancet Infect Dis 19(1):56-66

2. NORM/NORM-VET 2016. Usage of Antimicrobial agents and occurrence of antimicrobial resistance in Norway. Troms $\emptyset$ / Oslo 2017

3. NORM/NORM-VET 2008. Usage of antimicrobial agents and occurrence of antimicrobial resistance in Norway. Troms $\emptyset$ / Oslo 2009

4. Petersen A, Larssen KW, Gran FW, Enger H, Haeggman S, Makitalo B, Haraldsson G, Lindholm L, Vuopio J, Henius AE, Nielsen J, Larsen AR (2021) Increasing incidences and clonal diversity of methicillin-resistant Staphylococcus aureus in the Nordic countries - results from the Nordic MRSA surveillance. Front Microbiol 12:668900

5. Blomfeldt A, Hasan AA, Aamot HV (2016) Can MLVA differentiate among endemic-like MRSA isolates with identical spa-type in a low-prevalence region? PLoS One 11(2):e0148772

6. Uhlemann AC, Dumortier C, Hafer C, Taylor BS, Sanchez J, Rodriguez-Taveras C, Leon P, Rojas R, Olive C, Lowy FD (2012) Molecular characterization of Staphylococcus aureus from outpatients in the Caribbean reveals the presence of pandemic clones. Eur J Clin Microbiol Infect Dis 31(4):505-511

7. Bartels MD, Worning P, Andersen LP, Bes M, Enger H, Ås CG, Hansen TA, Holzknecht BJ, Larssen KW, Laurent F, Mäkitalo B, Pichon B, Svartström O, Westh H (2021) Repeated introduction and spread of the MRSA clone t304/ST6 in northern Europe. Clin Microbiol Infect 27(2):284.e1-284.e5. https://doi.org/10.1016/j. cmi.2020.05.004

8. Udo EE, Al-Lawati BA, Al-Muharmi Z, Thukral SS (2014) Genotyping of methicillin-resistant Staphylococcus aureus in the Sultan Qaboos University Hospital, Oman reveals the dominance of Panton-Valentine leucocidin-negative ST6-IV/t304 clone. New Microbes New Infect 2(4):100-105

9. Sonnevend A, Blair I, Alkaabi M, Jumaa P, Al Haj M, Ghazawi A, Akawi N, Jouhar FS, Hamadeh MB, Pal T (2012) Change in meticillin-resistant Staphylococcus aureus clones at a tertiary care 
hospital in the United Arab Emirates over a 5-year period. J Clin Pathol 65(2):178-182

10. Li D, Wu C, Wang Y, Fan R, Schwarz S, Zhang S (2015) Identification of multiresistance gene cfr in methicillin-resistant Staphylococcus aureus from pigs: plasmid location and integration into a staphylococcal cassette chromosome mec complex. Antimicrob Agents Chemother 59(6):3641-3644

11. Stegger M, Andersen PS, Kearns A, Pichon B, Holmes MA, Edwards G, Laurent F, Teale C, Skov R, Larsen AR (2012) Rapid detection, differentiation and typing of methicillin-resistant Staphylococcus aureus harbouring either mecA or the new mecA homologue mecA(LGA251). Clin Microbiol Infect 18(4):395-400

12. Harmsen D, Claus H, Witte W, Rothganger J, Claus H, Turnwald D, Vogel U (2003) Typing of methicillin-resistant Staphylococcus aureus in a university hospital setting by using novel software for spa repeat determination and database management. J Clin Microbiol 41(12):5442-5448

13. Mellmann A, Friedrich AW, Rosenkotter N, Rothganger J, Karch H, Reintjes R, Harmsen D (2006) Automated DNA sequencebased early warning system for the detection of methicillin-resistant Staphylococcus aureus outbreaks. PLoS Med 3(3):e33

14. Ridom spaServer. Ridom GmbH, https://spaserver.ridom.de/. Accessed 31 Dec 2016

15. Seemann T, Goncalves da Silva A, Bulach DM, Schultz MB, Kwong JC, Howden BP. Nullarbor, https://github.com/tseemann/ nullarbor. Accessed 13 Oct 2019

16. National Database of Antibiotic Resistant Organisms (NDARO). National Center for Biotechnology Information, https://www. ncbi.nlm.nih.gov/pathogens/antimicrobial-resistance/. Accessed 19 Apr 2020

17. Chen L, Yang J, Yu J, Yao Z, Sun L, Shen Y, Jin Q (2005) VFDB: a reference database for bacterial virulence factors. Nucleic Acids Res 33(Data issue):D325-328

18. Kaya H, Hasman H, Larsen J, Stegger M, Johannesen TB, Allesøe RL, Lemvigh CK, Aarestrup FM, Lund O, Larsen AR (2018) SCCmecFinder, a Web-Based Tool for Typing of Staphylococcal Cassette Chromosome mec in Staphylococcus aureus Using Whole-Genome Sequence Data. mSphere 3(1):e00612e00617. https://doi.org/10.1128/mSphere.00612-17

19. Page AJ, Cummins CA, Hunt M, Wong VK, Reuter S, Holden MT, Fookes M, Falush D, Keane JA, Parkhill J (2015) Roary: rapid large-scale prokaryote pan genome analysis. Bioinformatics 31(22):3691-3693

20. Price MN, Dehal PS, Arkin AP (2010) FastTree 2-approximately maximum-likelihood trees for large alignments. PLoS One 5(3):e9490

21. Kumar S, Stecher G, Li M, Knyaz C, Tamura K (2018) MEGA $\mathrm{X}$ : molecular evolutionary genetics analysis across computing platforms. Mol Biol Evol 35(6):1547-1549

22. Letunic I, Bork P (2021) Interactive Tree Of Life (iTOL) v5: an online tool for phylogenetic tree display and annotation. Nucleic Acids Res 49(W1):W293-W296. https://doi.org/10.1093/nar/ gkab301

23. Steen TW, Jorgensen SB, Garder KM, Kollerud Rdel R, Kvalvaag G, Blomfeldt A (2013) Cases of MRSA in nursing homes in Oslo 2005-11. Tidsskr Nor Laegeforen 133(17):1819-1823

24. Tegegne HA, Kolackova I, Florianova M, Gelbicova T, Wattiau P, Boland C, Karpiskova R (2019) Genome sequences of livestockassociated methicillin-resistant Staphylococcus aureus spa type t899 strains Belonging to three different sequence types (ST398, ST9, and ST4034). Microbiol Resour Announc 8 (2)

25. Bartels MD, Worning P, Andersen LP, Bes M, Enger H, As CG, Hansen TA, Holzknecht BJ, Larssen KW, Laurent F, Makitalo B, Pichon B, Svartstrom O, Westh H (2021) Repeated introduction and spread of the MRSA clone t304/ST6 in northern Europe. Clin Microbiol Infect 27(2):284 e281-284 e285

26. Kristinsdottir I, Haraldsson A, Thorkelsson T, Haraldsson G, Kristinsson KG, Larsen J, Larsen AR, Thors V (2019) MRSA outbreak in a tertiary neonatal intensive care unit in Iceland. Infect Dis (Lond) 51(11-12):815-823

27. Brown NM, Reacher M, Rice W, Roddick I, Reeve L, Verlander NQ, Broster S, Ogilvy-Stuart AL, D'Amore A, Ahluwalia J, Robinson S, Thaxter R, Moody C, Kearns A, Greatorex J, Martin H, Török ME, Enoch DA (2019) An outbreak of meticillin-resistant Staphylococcus aureus colonization in a neonatal intensive care unit: use of a case-control study to investigate and control it and lessons learnt. J Hosp Infect 103(1):35-43. https://doi.org/10. 1016/j.jhin.2019.05.009

Publisher's note Springer Nature remains neutral with regard to jurisdictional claims in published maps and institutional affiliations. 